\title{
Auch an das Seltene denken!
}

In der Pneumologie werden Erkrankungen subsumiert, die primär die Atemwege und/oder die Lunge betreffen. Dazu gehören aber auch Erkrankungen, in denen es mehr oder weniger große Überlappungen zu anderen Fachbereichen gibt oder bei denen die Lunge sekundär mitbetroffen ist. Dies betrifft insbesondere seltene Erkrankungen wie die zystische Fibrose (Überlappung mit der Pädiatrie), die pulmonalarterielle Hypertonie (Überlappung mit der Kardiologie), autoimmunologisch ausgelöste Lungenerkrankungen (Überlappung mit der Rheumatologie), oder die im Gegensatz zu den letzteren Erkrankungen häufiger vorkommenden Malignome der Lunge und des Rippenfells (Überlappung mit der Onkologie).

Immer mehr rücken viele der sog. „seltenen Lungenerkrankungen" selbst bei nicht wissenschaftlich interessierten Pneumologen in den Fokus, da die Zeiten der Blockbuster zu Ende geht. Als selten gilt eine Erkrankung in der Europäischen Union ab einer Prävalenz von < 1:2000 Personen [1]. Auch für seltene Erkrankungen stehen zunehmend Substanzen für die stationäre und ambulante Patientenversorgung zur Verfügung.

\section{0 seltene Erkrankungen mit Lungenbeteiligung}

ORPHANET verzeichnet über 90 seltene Erkrankungen, bei denen die Lunge mittelbar oder unmittelbar betroffen ist (www. orpha.net). Was vielleicht nur Wenige wissen: Jedes Jahr findet am letzten Februartag der „Rare Disease Day“ statt. Mit diesem Tag soll daran erinnert werden, dass in der Summe viele Menschen an einer der seltenen, aber sehr unterschiedlichen Erkrankungen leiden, und dass diese häufig schwer zu behandeln sind. Daher besteht ein großer Forschungsbedarf - vor allem in Bezug auf die pathophysiologischen Zusammenhänge - mit dem Ziel, neue Medikamente zu entwickeln [2].

Im Jahr 2016 hat das European Commission's Board of Member States 23 europäische Referenzgruppen (ERNs) einschließlich dem ERN-LUNG zugelassen [3, 4]. Ziel dieser Initiative ist es, das Wissen über seltene Erkrankungen zu bündeln, Zentren zu schaffen, die besondere Erfahrungen mit seltenen Lungenerkrankungen haben, Patienten zu registrieren und Empfehlungen $\mathrm{zu}$ verfassen.

Medizinische Einrichtungen können sich als Europäisches Referenzzentrum für seltene Lungenerkrankungen akkreditieren lassen. Derzeit besteht ERN-LUNG aus 60 Zentren in 12 Ländern und besitzt 9 Kerngruppen (Networks).

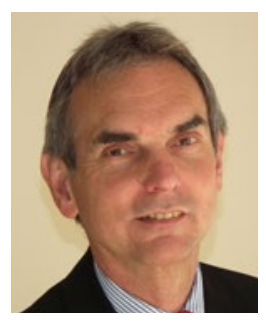

Prof. Dr. med. Adrian Gillissen

Kreiskliniken Reutlingen / Ermstalklinik

Med. Klinik III Innere Medizin/Pneumologie

Stuttgarter-Str. 100

72574 Reutlingen-Bad Urach

gillissen_a@klin-rt.de

Darüber hinaus sind natürlich Tagungen wichtig, auf denen alle Aspekte seltener Lungenerkrankungen vorgestellt und diskutiert werden. So fand das 7. International Meeting on Pulmonary Rare Diseases and Orphan Drugs Ende Februar in Mailand statt. Durch solche Veranstaltungen erfährt die Pneumologie eine zunehmende Differenzierung und Diversifizierung. Medien, die dieses immer komplexer werdende Wissen bündeln und vermitteln, werden daher immer bedeutungsvoller. Der/die pneumologisch interessierte Arzt/Ärztin ist trotz des vergleichsweise kleinen Fachs kaum in der Lage, selbst die bedeutungsvollen Publikationen zeitaktuell zu lesen und im Detail zu kennen. Dies betrifft vor allem die selteneren Erkrankungen, zu denen z. B. auch Pilzinfektionen in der Lunge gehören, die in einem Fortbildungsbeitrag dieser Ausgabe thematisiert werden $(\triangleright$ s. S. 37).

Somit erwartet den Leser auch in dieser Ausgabe der PneumoNews wieder ein buntes und interessantes Potpourri an neuem Wissen auf dem Gebiet der Pneumologie.

\section{Literatur}

1. Rare Diseases Europe (EURORDIS). What is rare disease day? www.rarediseaseday.org/article/what-is-rare-disease-day Date last accessed: December 262016.

2. Harari S, Lau EMT, Tamura Y, et al. Rare (Pulmonary) Disease Day: " Feeding the breath, Energy for life!" Eur Respir J 2015; 45: 297-300.

3. Humbert M., Wagner T.O.F. Rare respiratory diseases are ready for primetime: from Rare Disease Day to the European Reference Networks. European Respiratory Journal 2017; 49(2)1602232; DOI: $10.1183 / 13993003.02232-2016$

4. The European Commission Directorate of Health and Food Safety. European reference networks: call for applications 2016. http://ec.europa.eu/ health/ern/implementation/call_en Date last accessed: Dec 262016 